\title{
Control of T-DNA Transfer from Agrobacterium tumefaciens to Plants Based on an Inducible Bacterial Toxin-Antitoxin System
}

\author{
Erna Denkovskienè,,${ }^{1,2,+}$ Šarūnas Paškevičius, ${ }^{1,2}$ Justina Stankevičiūtè, ${ }^{1}$ Yuri Gleba, ${ }^{3}$ and \\ Aušra Ražanskiené ${ }^{1}$ \\ ${ }^{1}$ Nomads UAB, Geležinio vilko 29A, LT-01112, Vilnius, Lithuania \\ ${ }^{2}$ Vilnius University, Institute of Biotechnology, Saulètekio al. 7, LT-10257 Vilnius, Lithuania \\ ${ }^{3}$ Nomad Bioscience GmbH, Biozentrum Halle, Weinbergweg 22, D-06120 Halle (Saale), Germany
}

Accepted 20 June 2020.

\begin{abstract}
High-value pharmaceutical products are already successfully produced in contained facilities using Agrobacterium-mediated transient transformation of plants. However, transfection methods suitable for open field applications are still desirable as a cheaper alternative. Biosafety concerns related to the use of recombinant agrobacteria in an industrial transfection process include possible transformation or transfection of unintended hosts or spread of the genetically modified agrobacteria in the environment. In this paper, we explored a novel biocontrol approach resulting in greater biosafety of the transient expression process in plants. Our proposed solution involves inducible expression of Agrobacterium tumefaciens toxin PemK and antitoxin PemI that provides for strictly regulated T-DNA transfer from agrobacteria to plants. We also identified several other toxins from putative Agrobacterium toxin-antitoxin modules and demonstrate their potential usefulness in the control of Agrobacterium tumefaciens as a DNA vector.
\end{abstract}

Keywords: biocontrol, toxin-antitoxin system, transient expression

Agrobacterium tumefaciens, a soil plant-pathogenic bacterium, has become a very powerful DNA vector widely used in development of genetically modified plants. It is also used for transient expression of pharmaceutical and nonpharmaceutical proteins in Nicotiana benthamiana, tobacco, lettuce, tomato, and other plant species (Krenek et al. 2015; Schulz et al. 2015; Tschofen et al. 2016; Yao et al. 2015). Transient production of high-value therapeutic proteins in plants in contained facilities is increasingly applicable also in large-scale production such as a Nicotiana benthamiana-based influenza vaccine manufacturing by the Medicago company (Hodgins et al. 2019). However, transfection methods suitable for field applications are still desirable for the industrial-scale production of cost-sensitive products, such as the low-caloric sweetener thaumatin, for the partial substitution of the sugar in food, or cellulases produced

\section{${ }^{\dagger}$ Corresponding author: E. Denkovskienė; erna@nomadsbio.lt}

*The $\boldsymbol{e}$-Xtra logo stands for "electronic extra" and indicates there are supplementary figures and tables published online.

The author(s) declare no conflict of interest.

๑) 2020 The American Phytopathological Society for the conversion of cellulosic biomass into ethanol as a fuel extender (Hahn et al. 2015; Szwacka et al. 2012; Tusé et al. 2014). Outdoor cultivation is superior to that in contained facilities because of lower upstream process capital investments required for plant biomass production, no need for substrate or nutrient solution management, and other factors. However, the release of genetically modified Agrobacterium strains for plant transfection into the open environment causes potential biosafety issues. There are several approaches being investigated for the biocontainment of genetically modified microorganisms, including multiple metabolic or synthetic auxotrophy or synthetic gene circuits. However, none of them can ensure complete biosafety because of specific or universal disadvantages, such as function regain because of horizontal gene transfer or biocontainment system disruption because of mutagenesis (Lee et al. 2018). Thus, only a combination of different strategies can help to reach the goal of the minimum risk. Earlier, we have demonstrated that efficient control of T-DNA transfer from recombinant Agrobacterium spp. to plants can be achieved by engineering inducible expression of agrobacterial virulence gene virE2 (Denkovskiene et al. 2015). The goal of our recent research was to explore the possibility of constructing a more biosafe transient expression system for preventing unintended T-DNA transfer by using the potential of natural bacterial toxin-antitoxin (TA) modules.

In eukaryotes, the classical form of programmed cell death is apoptosis (Fadeel and Orrenius 2005). Many recent studies have shown that particular mechanisms of programmed cell death also exist in prokaryotes, allowing them to withstand environmental stresses, such as nutrient deprivation, antibiotics exposure, and more (Hu et al. 2010). For most eubacteria and archaea, the best-known mechanisms involved in bacterial programmed cell death are TA systems. TA systems function in bacterial stress tolerance, virulence, phage defense, and also biofilm formation (Wang and Wood 2011). All TA systems are normally composed of two elements, a toxin and an antitoxin. Basically, the toxin suppresses bacterial growth and exists as a stable protein, while the antitoxin is a labile protein or RNA that neutralizes the toxin (Yamaguchi and Inouye 2016). TA systems have been categorized into seven types according to the mechanism by which the antitoxin interacts with the toxin. In most cases, toxin and antitoxin genes are organized into operons. The expression of a TA operon is autoregulated by the antitoxin at the transcription level, while the toxin acts as a corepressor (Christensen et al. 2004). In normal conditions in type II and type III systems, the antitoxin blocks toxin action 
by tight binding (Goeders and Van Melderen 2013; Sala et al. 2014). However, when a microorganism experiences nutrient deficiency, antibiotic treatment, environmental stress, immune system response, bacteriophage infection, or oxidative stress, the concentration of antitoxin may decrease and the free toxin suppresses bacterial growth or causes cell death. Toxins interfere with membrane integrity preventing proton movement (Wang et al. 2012; Weel-Sneve et al. 2013), act as endoribonucleases, or disturb DNA replication and translation by inhibiting DNA gyrase (Yamaguchi and Inouye 2016). A small portion of known toxins stop cell division by interfering with the synthesis of bacterial peptidoglycan (Mutschler and Meinhart 2011) or inhibiting the polymerization of bacterial cytoskeletal proteins (Tan et al. 2010).

We identified several putative toxins, homologs of toxins from TA modules in the Agrobacterium genome and demonstrated the ability of these toxins to cause bacterial death when expressed ectopically. We used one TA pair, pemIK, for the construction of a transient expression system with strictly regulated T-DNA transfer.

\section{RESULTS}

\section{Identification of A. tumefaciens TA modules.}

The preliminary screening of putative TA loci in the $A$. tumefaciens C58 genome was performed with the webbased tool RASTA-Bacteria. All open reading frames found by the RASTA-Bacteria webtool in a provided sequence, whether annotated or not, undergo a scoring process to determine their likelihood to belong to the TA family of genes, according to several criteria settled by the authors (Sevin and Barloy-Hubler 2007), and are ranked accordingly in a given list of candidate TA systems. Scores above $70 \%$ indicate high probability of an active TA module, while lower scored candidates require further manual verification. A RASTA-Bacteria cut-off score $>47 \%$ was manually settled and TA systems were selected - three from the circular chromosome, one from the linear chromosome, and one from the Ti plasmid. Additionally, the ietS toxin from the Ti plasmid was selected based on data by Yamamoto et al. (2009) (Table 1). The Iets gene was also included among potential TA systems provided by RASTA-Bacteria (as Atu6083), however, it scored only 27\% and was not recognized as a candidate toxin initially.

\section{Ectopically expressed putative toxins are capable of causing Agrobacterium death.}

All cloned toxin genes were placed under the control of strong and tightly regulated cumic acid-inducible promoters and constructed binary vectors were transformed into the A. tumefaciens GV3101 strain (constructed strains are listed in Table 2). The resultant strains were used to investigate the toxic effect of cloned putative toxins. The expression of Agrobacterium toxins PemK, Dead, VapC, PIN, and IetS reduced bacterial colony-forming unit (CFU) counts by a very similar range, 2.7 to 3.8 orders of magnitude, after $4 \mathrm{~h}$ of induction with $150 \mu \mathrm{M}$ cumic acid in liquid medium. By contrast, the nuclease-like toxin demonstrated a much weaker activity against $A$. tumefaciens, reducing CFU count by only 0.5 order of magnitude (Fig. 1). Thus, we demonstrated that five of cloned toxins are capable of killing agrobacteria efficiently while expressed ectopically.

\section{PemK effectively supresses agrobacteria-based plant transient expression.}

As the next step, we investigated whether toxins inserted under cumic acid-regulated promoters could be used to suppress the agrobacteria-based transient expression system when bacteria are sprayed onto Nicotiana benthamiana plants. As pemK was the first toxin cloned, it was used for the construction of the model system.

Vector pNMDV371 ( $\mathrm{P}_{T 5 / \mathrm{CuO}}:$ :pemK) was cotransformed into Agrobacterium tumefaciens with pICH27566, a PVX (potato virus $\mathrm{X}$ )-based binary expression vector for the expression of green fluorescent protein (GFP) in plants (Supplementary Fig. $\mathrm{S} 1$ ). As we demonstrated previously, transient expression assays in $N$. benthamiana following agroinfiltration with A. tumefaciens GV3101 (pICH27566) show strong GFP fluorescence in plant leaves 4 to 5 days postinfiltration. In these assays, GFP expression foci, as they are a phenotypic result of the T-DNA transfer, were calculated on plant leaves (Denkovskienè et al. 2015). Two Agrobacterium strains, control strain GV3101 (pICH27566) and GV3101 (pICH27566, pNMDV371), containing the $\mathrm{P}_{T 5 / \mathrm{CuO}}:$ : pem $K$ construct in addition to the GFP-expression vector, were used for agrospray experiments. To induce pemK expression in the agrobacterium, $150 \mu \mathrm{M}$ of cumic acid was added into the spraying solution. Seven days after the spraying, both GFP foci counts on plant leaves and Agrobacterium CFU counts in the same leaves were evaluated. CFU counts of viable agrobacteria 7 days after spraying demonstrated significant differences. A total of $4.1 \log _{10}$ of bacteria were retrieved from a gram of fresh plant leaves sprayed with the control strain (pICH27566). By sharp contrast, no bacteria were retrieved from the cumic acid-induced strain containing the $\mathrm{P}_{T 5 / \mathrm{CuO}}:$ :pemK cassette (pNMDV371, pICH27566). Even without induction by cumic acid, the toxin expression cassette-harboring strain demonstrated CFU counts almost 100-fold lower than the control

Table 1. Toxins from Agrobacterium tumefaciens (C58) genome toxin-antitoxin (TA) systems ${ }^{\mathrm{a}}$

\begin{tabular}{|c|c|c|c|}
\hline $\begin{array}{l}\text { Putative TA module } \\
\text { or toxin alone }\end{array}$ & Locus tags & Location in the genome & Predicted function of the toxin and related references \\
\hline pemIK & Atu0939-Atu0940 & Circular chromosome AE007869.2 & $\begin{array}{l}\text { Originally genes of pem (plasmid emergency maintenance) were } \\
\text { found in E. coli plasmid R100 (Tsuchimoto et al. 1992). Has } \\
\text { endoribonuclease activity (Lee et al. 2012) }\end{array}$ \\
\hline $\operatorname{vapC}$ & Atu 1004 & Circular chromosome AE007869.2 & $\begin{array}{l}\text { VapC family toxin from type II TA systems. VapC family members are } \\
\text { thought to be site-specific endoribonucleases that cleave tRNA (fMet) } \\
\text { in the anticodon stem-loop between nucleotides }+38 \text { and }+39 \text { in vivo } \\
\text { and in vitro (Winther and Gerdes } 2011 \text {; Winther et al. 2016) }\end{array}$ \\
\hline PIN & Atu2140 & Circular chromosome AE007869.2 & $\begin{array}{l}\text { VapC family toxin from type II TA system. Functions as ribonuclease, } \\
\text { contains the PIN domain (Arcus et al. 2011) }\end{array}$ \\
\hline dead & Atu3013 & Linear chromosome AE007870.2 & $\begin{array}{l}\text { VapC family toxin. Predicted nucleic acid binding protein, contains the } \\
\text { PIN domain }\end{array}$ \\
\hline Nuclease-like & Atu6122 & Ti plasmid AE007871.2 & $\begin{array}{l}\text { Thermonuclease family protein, part of traCDG operon regulated by } \\
\text { quorum sensing, gene named } y c i \text { (Cho and Winans 2007) }\end{array}$ \\
\hline ietS & Atu6083 & Ti plasmid AE007871.2 & Serine protease function, plasmid stabilization (Yamamoto et al. 2009) \\
\hline
\end{tabular}

a The RASTA-Bacteria tool was used to search for TA systems in the Agrobacterium genome. 
strain and reached $2.2 \log _{10}$ per gram of fresh plant leaf (Fig. $2 \mathrm{~A})$. We presume that this reduction in the number of bacteria resulted from the basal activity of the $\mathrm{P}_{T 5 / \mathrm{CuO}}$ promoter. A growth curve of uninduced $\mathrm{P}_{T 5 / C u O}:$ :pemK strain showed similar growth compared with control strain harboring $\mathrm{P}_{T 5 / \mathrm{CuO}}:$ : $l a c Z$ in a period of $24 \mathrm{~h}$ (Supplementary Fig. S4); however, the effect of a basal expression of pemK may accumulate in a longer period of time, as CFU counts of Agrobacterium colonies was evaluated after 7 days post-agrospray in our experiment. Count of GFP expression foci was similar between the control strain and the uninduced $\mathrm{P}_{T 5 / \mathrm{CuO}}:$ :pemK cassettecontaining strain, with the numbers of GFP foci per leaf of $2.8 \log _{10}$ and $2.7 \log _{10}$, respectively. The cumic acid-induced
$\mathrm{P}_{\text {T5/CuO }}:$ :pemK cassette-containing strain demonstrated strongly reduced T-DNA transfer $\left(1 \log _{10}\right)$ (Fig. 2B). Thus, the induced expression of toxin pem $K$ efficiently killed recombinant agrobacteria and most of the killing seemed to occur before the T-DNA transfer took place, as demonstrated by reduced GFP foci numbers.

\section{Construction of a regulated T-DNA transfer system and evaluation of induction kinetics of $\mathbf{P}_{\text {virE }}$ and cumic acid-regulated promoters.}

In an ideal regulated T-DNA transfer system, the bacteria should be able to transfer the T-DNA to plants in a programmed timely manner, for example, just one or two days after plant

Table 2. List of Agrobacterium tumefaciens strains used in this study

\begin{tabular}{|c|c|c|}
\hline Strain & Description & References \\
\hline GV3101 & (pMP90RK), nopaline, Rif $^{r}$ & Koncz and Schell 1986 \\
\hline GV3101_pemIK & (pMP90RK), nopaline, Rif $^{\mathrm{r}}$, pemIK region deleted & This study \\
\hline ICH011 & GV3101_virE2 (pMP90RK), nopaline, Rif $^{\mathrm{r}}$ & Denkovskiené et al. 2015 \\
\hline ICH011 (pNMDV386) & Contains pICH27566-cymR, $\mathrm{P}_{T 5 / C u O}:: v i r E 2$ & Denkovskiené et al. 2015 \\
\hline ICH011 (pNMDV356) & Contains pICH27566-cymR, $\mathrm{P}_{\text {tac/CuO }}::$ virE2 & Denkovskiené et al. 2015 \\
\hline GV3101 (pICH27566) & Contains potato virus $\mathrm{X}$-based vector with green fluorescent protein & Icon Genetics \\
\hline GV3101 (pNMDV621) & Contains pNDC-cymR-P tac/CuO ::dead & This study \\
\hline GV3101 (pNMDV622) & Contains pNDC-cymR-P $\mathrm{P}_{t a c / C u O}:: P I N$ & This study \\
\hline GV3101 (pNMDV623) & Contains pNDC-cymR-P & This study \\
\hline GV3101 (pNMDV624) & Contains pNDC-cymR-P $\mathrm{P}_{t a c / C u O}:$ :nuclease_like & This study \\
\hline GV3101 (pNMDV371) & Contains pNDC-cymR-P $\mathrm{P}_{T 5 / \mathrm{CuO}}::$ pemK-P $P_{\text {tetR }}$ & This study \\
\hline GV3101 (pNMDV350) & Contains pNDC-cymR-P ${ }_{T 5 / \mathrm{CuO}}::$ lacZ- $P_{\text {tetR }}$ & This study \\
\hline GV3101 (pNMDV375) & Contains pNDC-cymR-P ${ }_{T 5 / \mathrm{CuO}}::$ pemIK & This study \\
\hline GV3101 (pNMDV536) & Contains pNDC-cymR-P ${ }_{t a c / C u O}:: i e t S$ & This study \\
\hline GV3101 (pNMDV371+pICH27566) & Contains pNDC-cymR-P $\mathrm{P}_{T 5 / \mathrm{CuO}}::$ pemK-P $P_{\text {tetR }}+$ pICH27566 & This study \\
\hline GV3101_pemIK (pNMDV515+pNMDV535) & Contains pNDC-P ${ }_{\text {virE }}:$ pemK + pICH27566-cymR-P tac/CuO $:: p e m I$ & This study \\
\hline GV3101 (pNMDV535) & Contains pICH27566-cymR-P tac/Cuo::pemI & This study \\
\hline
\end{tabular}

5

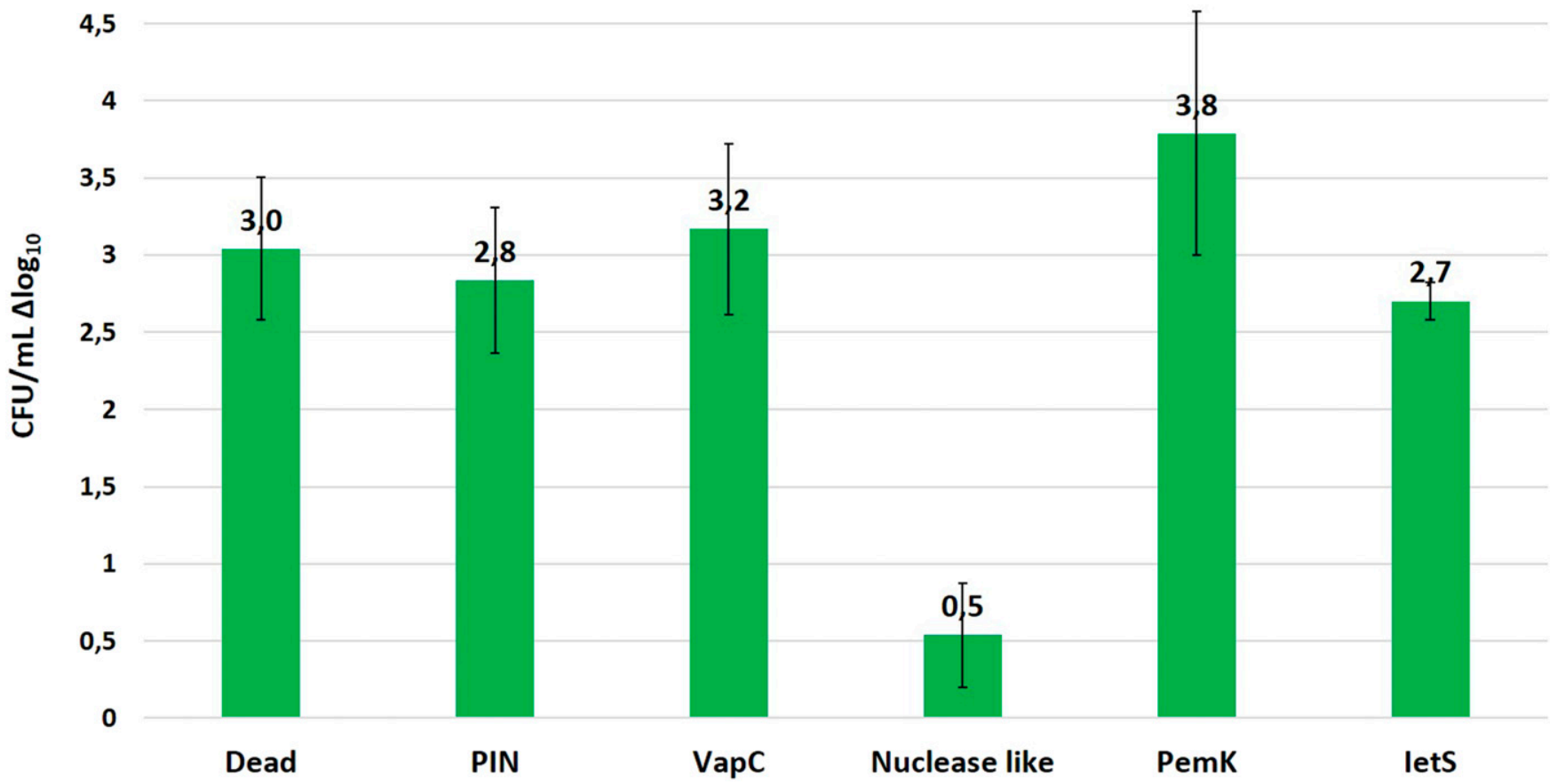

Fig. 1. The ability of ectopically expressed toxins to kill Agrobacterium tumefaciens. The overnight cultures of A. tumefaciens GV3101 with plasmids carrying toxin expression cassettes were diluted to an optical density at $595 \mathrm{~nm}$ approximately 0.1 to 0.2 . Each diluted culture was split in two. The medium with $150 \mu \mathrm{M}$ cumic acid and the inducer-free medium. The cultures were further incubated at $28^{\circ} \mathrm{C}$ and $220 \mathrm{rpm}$ for $4 \mathrm{~h}$, then, serial dilutions were made and were plated on selective Luria Bertani-agar medium without inducer $\left(25 \mu \mathrm{l}\right.$ of each culture). Colony-forming units (CFU) were counted after $48 \mathrm{~h}$ of incubation at $28^{\circ} \mathrm{C}$. The effectiveness of each toxin was evaluated by using the formula $\Delta \log _{10}=\log _{10}$ (CFU per milliliter of noninduced culture) - $\log _{10}$ (CFU per milliliter of induced culture). Data are the mean \pm standard deviation of three independent experiments. 
transformation, and then lose this ability. We have demonstrated that cumic acid-induced pemK expression kills bacteria too rapidly, before the T-DNA transfer fully takes place. Thus, to give time for agrobacteria to transfer the T-DNA, either the pemK expression or its action should be delayed.

The PemIK TA module belongs to type II TA systems. In type II systems, the antitoxin, which is a labile protein, blocks the protein toxin action by tight binding (Goeders and Van Melderen 2013; Sala et al. 2014). Thus, including pemI into our transient expression system seems appropriate for delaying the toxic effect of PemK activity. To obtain a tightly regulated system, we aimed to express both the toxin and the antitoxin from the separate promoters with different regulation profiles. We sought an early expression of pemI antitoxin and a delayed but strong expression of the pemK toxin.

In the quest for an appropriate promoter pair, we compared the expression kinetics of a native $\mathrm{P}_{\text {virE }}$ promoter of Agrobacterium sp. strain GV3101 and two cumic acid-inducible promoters $\mathrm{P}_{T 5 / C u O}$ and $\mathrm{P}_{t a c / C u O}$ (Denkovskiene et al. 2015). $\mathrm{P}_{\text {virE }}$ is induced by plant phenolic compounds released during pathogen-plant interaction. $\mathrm{P}_{\text {vire }}$ induction begins the transcription of genes from virE operon, and the T-DNA transfer chain of events is thus initiated (McCullen and Binns 2006).

Three Agrobacterium strains, the first harboring $\mathrm{P}_{T 5 / \mathrm{CuO}}:$ : virE2 [ICH011 (pNMDV386)], the second harboring $\mathrm{P}_{t a c / C u O}:$ : virE2 [ICH011 (pNMDV356)], and the GV3101 strain containing vector $\mathrm{pICH} 27566$ were used for plant infiltration. The infiltrated leaves were collected at $4,24,48$, and $72 \mathrm{~h}$ postinfiltration, were frozen in liquid nitrogen, and were subjected to RNA extraction. The virE2 expression analysis by real-time PCR revealed different expression kinetics from synthetic cumic acid-regulated promoters and a natural virE promoter. At $4 \mathrm{~h}$ postinfiltration, the first timepoint investigated, virE2 expression from cumic acid-induced $\mathrm{P}_{T 5 / C u O}$ and $\mathrm{P}_{t a c / C u O}$ was already almost five times higher than the basal expression from uninduced promoters. By this time, the expression from the native virE promoter was still very low and comparable to the expression from uninduced $\mathrm{P}_{T 5 / C u O}$ and $\mathrm{P}_{t a c / C u O}$. However, at $24 \mathrm{~h}$ postinfiltration, $\mathrm{P}_{\text {virE }}$-driven virE2 expression is multiplied sevenfold and is similar to the expression from $\mathrm{P}_{T 5 / C u O}$ and $\mathrm{P}_{\text {tac } / \mathrm{CuO}}$. At 48 and $72 \mathrm{~h}$ postinfiltration, the relative expression from $\mathrm{P}_{\text {virE }}$ is equal to or higher than the expression from cumic acid-inducible promoters. VirE2 expression from all three promoters reached its peak after $48 \mathrm{~h}$ and then started to decrease, demonstrating a two times lower relative expression level after $72 \mathrm{~h}$ (Fig. 3).
We presumed that if the antitoxin is expressed before the toxin and accumulated in the cell in a certain amount before the expression of toxin starts, it will be able to inactivate the toxin and to allow bacteria to survive for the time lapse sufficient for the T-DNA transfer. As the natural induction of $\mathrm{P}_{\text {virE }}$ is delayed in comparison with the chemical induction of $\mathrm{P}_{t a c / C u O}$, we inserted the antitoxin pemI under the $\mathrm{P}_{t a c / C u O}$ promoter and the toxin pemK under the control of $\mathrm{P}_{\text {vire }}$ of Agrobacterium sp. strain KYRT-1, described in our previous publication (Denkovskiene et al. 2015). The $\mathrm{P}_{t a c / C u O}:$ :pemI was inserted into the backbone of the PVX-based GFP expression vector pICH27566, and $\mathrm{P}_{\text {virE }}:$ :pemK $K$ was cloned in a separate plasmid of a different incompatibility group (Supplementary Fig. S2). Both vectors were transformed into GV31014pemIK strain, to avoid any interference with the native pemIK module expressed from the Agrobacterium genome. The strain obtained $\left(\mathrm{P}_{\text {vire }}:\right.$ : pemK $; \mathrm{P}_{\text {tac } / \mathrm{CuO}}::$ pemI $)$ was used for agroinfiltration experiments and T-DNA transfer evaluation by counting the GFP foci. Practically no T-DNA transfer was observed with the Agrobacterium strain harboring both pemK and pemI constructs when no cumic acid was added (four GFP foci per leaf, on average). When Agrobacterium suspension contained cumic acid for the induction of pemI, the T-DNA transfer was reconstituted; 394 GFP foci per leaf were obtained after induction with $50 \mu \mathrm{M}$ cumic acid, and 759 GFP foci per leaf after induction with $150 \mu \mathrm{M}$ cumic acid (Fig. 4). For comparison, infiltration with the strain containing only the $\mathrm{P}_{t a c / C u O}:$ :pemI construct resulted in 667 GFP foci per leaf, meaning that the induced T-DNA transfer was similar or even more efficient than the wild-type T-DNA transfer (115.3\%).

Toxin expression from the $\mathrm{P}_{\text {vire }}$ promoter does not significantly reduce the number of bacteria (not shown). We presume that only a minor fraction of bacteria in contact with the plant leaf will start the T-DNA transfer events cascade and undergo virE promoter induction. Most bacteria left on the leaf surface after infiltration will not be induced for the T-DNA transfer event cascade and, thus, will not be affected by the toxic effect of PemK. This contrasts with $\mathrm{P}_{t a c / C u O}$ or $\mathrm{P}_{T 5 / \mathrm{CuO}}$, which are induced by cumic acid despite the induction state of their virulence genes.

In summary, we achieved tight regulation in a plant transient expression system, based on the expression of the toxin pemK under control of the native plant virE promoter and expression of the pemI antitoxin from a chemically inducible $\mathrm{P}_{t a c / C u O}$ promoter. In the proposed system, the T-DNA transfer is possible only for a short time after agroinfiltration, while
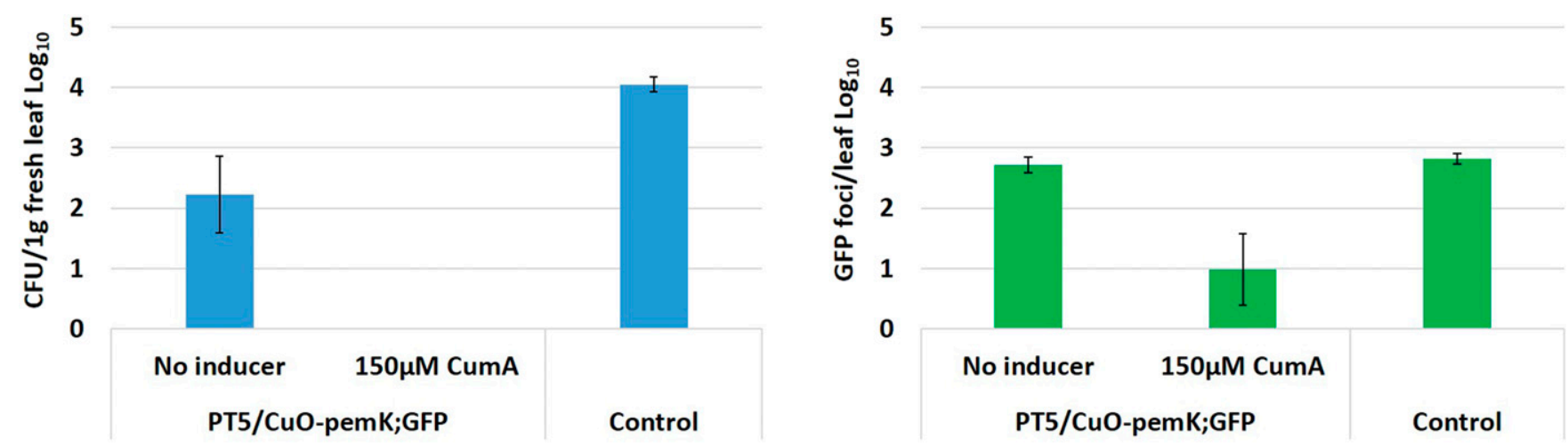

Fig. 2. Evaluation of toxin PemK activity against Agrobacterium tumefaciens and green fluorescent protein (GFP) expression foci number in agrospray experiments. Nicotiana benthamiana plants were sprayed with cumic acid-induced or noninduced $\mathrm{P}_{T 5 / C u O}:: p e m K$ construct-containing strain $\mathrm{GV} 3101$ (pICH27566, pNMDV371) and with control strain GV3101 (pICH27566). GFP loci were counted on sprayed leaves 7 days after spraying. Next, the same leaves were ground in liquid nitrogen for agrobacteria isolation and counting colony-forming units (CFU). The amount of bacteria per gram of fresh leaves was calculated. Data are the mean \pm standard deviation of three independent experiments. 
pemI expression is induced by cumic acid, present in the agroinfiltration solution. When the inductor is depleted, pemI expression stops and the T-DNA transfer becomes impossible as bacteria with induced virulence genes are simultaneously induced for transcription of the pemK toxin. Every subsequent T-DNA transfer attempt from the bacteria remaining in the soil should lead to the death of bacteria upon induction of the virE promoter.

\section{DISCUSSION}

The main goal of this work was to create a tightly controlled T-DNA transfer system for large-scale applications of genetically modified agrobacterium in agrobiotechnology, such as those requiring the release of genetically modified agrobacteria into an open environment. In our previous study, we described the construction of a regulated transient expression system by deleting the A. tumefaciens virE2 gene essential for T-DNA transfer and placing it under different cumic acid-inducible promoters. In that system, agrobacteria were able to transfer T-DNA to plants only when cumic acid was present in the spraying solution. After depletion of the inductor, no T-DNA transfer was possible, except for very rare events, probably explained by the residual activity of promoters (Denkovskiene et al. 2015). Our previously constructed T-DNA transfer regulation system may be further supplemented with a TA-based expression system, thus minimizing unintended T-DNA spread. Alternatively, this proposed mechanism is useful in cases when an appropriate virE2-deficient Agrobacterium strain is not available.
Bacterial TA systems seemed to be a priori attractive, however challenging, tools for our purposes because of limited understanding of the upstream and downstream regulation of TA systems and the scarcity of specific knowledge about Agrobacterium TA systems. Toxins from bacterial TA modules have already been successfully used for the construction of biocontainment systems. In particular, bacterial TA systems were shown to work in yeast containment systems. Escherichia coli RelBE and Kis-Kid (homolog of pemI-pemK) TA modules have been introduced into genetically modified Saccharomyces cerevisiae (de la Cueva-Méndez et al. 2003; Kristoffersen et al. 2000). The Kis-Kid controlled expression system was engineered using a strategy similar to ours, with the toxin and antitoxin being inserted under the control of two different inducible promoters, Met 25 and CUP1. Yeast was transformed with the plasmid in which kis (antitoxin) expression is repressed in the presence of methionine and kid (toxin) expression is activated in the presence of $\mathrm{Cu}^{2+}$. CFU count of yeast was lowered by five orders of magnitude in the presence of methionine and $\mathrm{Cu}^{2+}$, with no growth inhibition detected in the absence of both inducers or in the presence of $\mathrm{Cu}^{2+}$ only. Coexpression of the antitoxin together with the toxin protected yeast from growth inhibition (de la Cueva-Méndez et al. 2003). Later, an alternative containment system for $E$. coli ('Deadman') based on toxins $m a z F$ and $c c d B$ was constructed (Chan et al. 2016). These works demonstrated the high potential of bacterial TA systems for biocontainment of prokaryotes, lower eukaryotes, or even for cell ablation-based strategies for controlling the spread of genetically modified plants (expression of yoeB toxin from Streptococcus

25

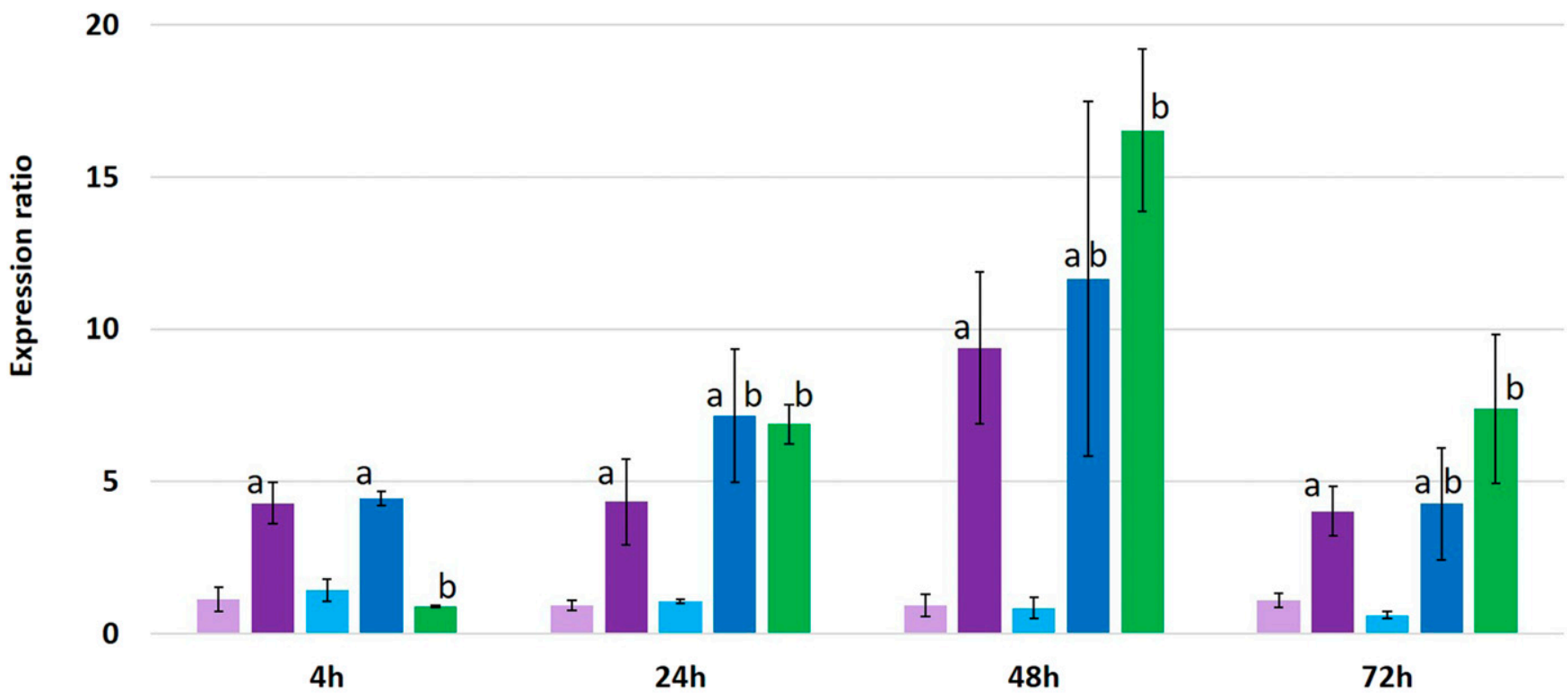

PT5/CuO Un $\square$ PT5/CuO In $\square$ Ptac/CuO Un $\square$ Ptac/CuO In $\square$ PvirE

Fig. 3. Reverse transcription-quantitative PCR (RT-qPCR) analysis of virE2 expression from cumic acid inducible promoters $\mathrm{P}_{T 5 / C u O}$ and $\mathrm{P}_{t a c / C u O}$ and from the

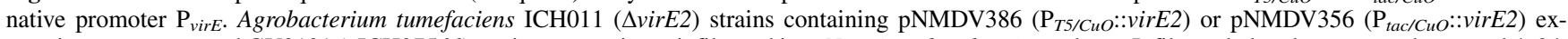
pression constructs, and GV3101 (pICH27566) strain were syringe- infiltrated into Nicotiana benthamiana plants. Infiltrated plant leaves were harvested 4, 24, 48 , and $72 \mathrm{~h}$ after agroinfiltration. The expression ratio of virE2 was evaluated by RT-qPCR. Un $=v i r E 2$ expression from uninduced $\mathrm{P}_{T 5 / C u O}$ and $\mathrm{P}_{t a c} / C u O$ promoters, In $=$ virE2 expression from $150 \mu \mathrm{M}$ cumic acid-induced $\mathrm{P}_{T 5 / C u O}$ and $\mathrm{P}_{\text {tac } / C u O}$ promoters, $\mathrm{P}_{\text {virE }}=$ virE2 expression from wild-type $\mathrm{P}_{\text {virE }}$ promoter in $\mathrm{GV} 3101$. Data are the mean \pm standard deviation of three independent experiments. Unpaired $t$ test was used for statistical analysis. Lower case a and b indicate significance of difference between values. Values are not significantly different within group a or b but are significantly different between groups. 
pneumoniae TA module yefM-yoeB, shown to be lethal in Arabidopsis [Bakar Yeo and Harikrishna 2015]).

Examples of successful application of TA systems in other microorganisms encouraged us to test whether TA systems could be suitable as a means of control of T-DNA transfer from genetically modified agrobacteria. We provide, here, an efficient inducible expression system based on the action of the bacterial TA system utilizing a type II TA system, pemIK. In a previous study, we constructed and analyzed several inducible promoters to be used in A. tumefaciens (Denkovskiene et al. 2015). Cumic acid-inducible promoters $\mathrm{P}_{t a c / C u O}, \mathrm{P}_{T 5 / C u O}$, $\mathrm{P}_{\text {lacUV5/CuO }}$, and $\mathrm{P}_{\text {virE/CuO }}$ demonstrated different expression levels and strict regulation by the inducer in Agrobacterium tumefaciens. We now used these data for the choice of a promoter pair for toxin pemK and antitoxin pemI expression. Pathogen-plant interaction induced expression of the toxin pemK from a natural Agrobacterium promoter, $\mathrm{P}_{\text {vire }}$, and the expression of the antitoxin pemI from a cumic acid inducible promoter, $\mathrm{P}_{t a c / C u O}$, resulted in efficient transient transfection of $N$. benthamiana plants after 6 days post-agroinfiltration. T-DNA transfer was possible only for a short time after agroinfiltration, while cumic acid, the inductor of expression of the antitoxin, is present. When the inductor is depleted, the TDNA transfer becomes impossible as bacteria with induced virulence genes were equally induced for transcription of the pemK toxin. Any subsequent T-DNA transfer from bacteria remaining in the soil after agroapplication (in the absence of the chemical inducer) should lead to the death of bacteria. Controlled T-DNA transfer would be beneficial in combination with other safeguard approaches for biocontainment, for example, multiple auxotrophy or genome recoding (Lajoie et al. 2013; Mandell et al. 2015). We used plasmid-expressed pemI and pemK in our experimental system, but, for further improvement, both expression cassettes should be integrated into the Agrobacterium genome. We also identified and evaluated, for the first time, activity of several other A. tumefaciens toxins from TA systems. Toxins Dead, PIN, VapC, and IetS were all highly efficient in reducing Agrobacterium CFU count by 2.7 to 3.8 orders of magnitude after a 4-h incubation with an inducer.

As with any biosafety or biocontainment technology applied for microorganisms, we can never expect a zero risk of biosafety system disruption because of horizontal gene transfer or

1200

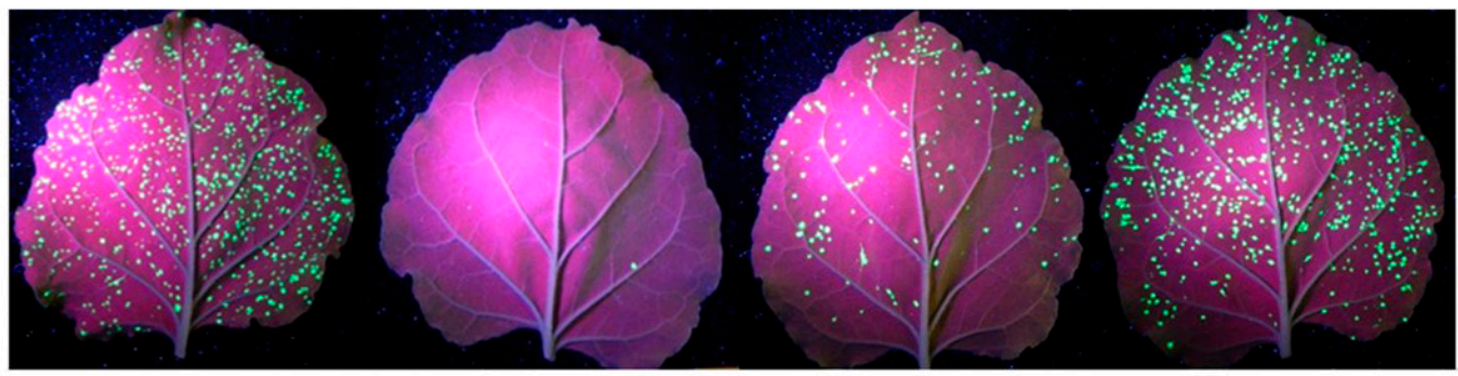

1000

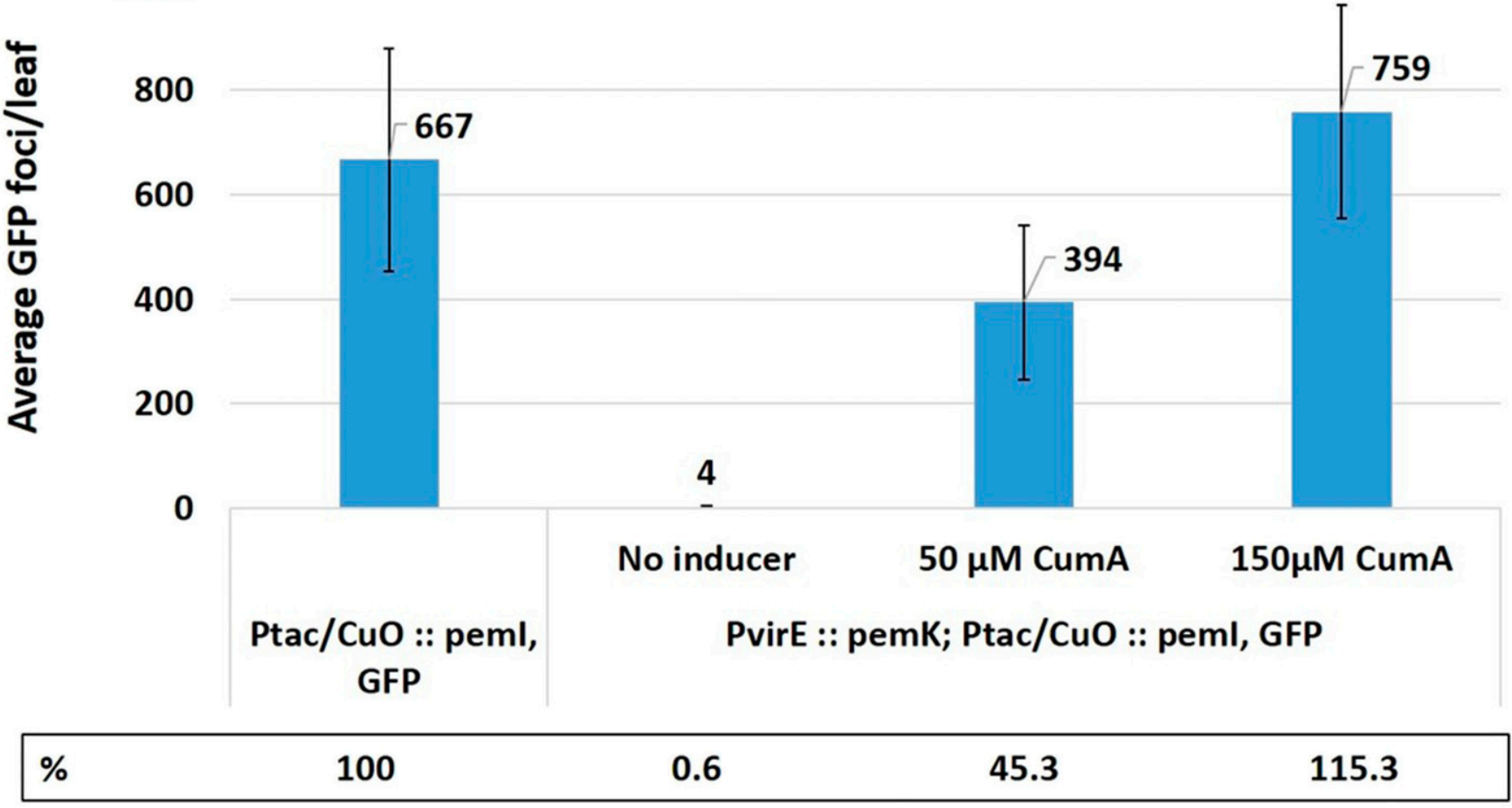

Fig. 4. Evaluation of green fluorescent protein (GFP) expression foci number after agroinfiltration with an Agrobacterium strain harboring $\mathrm{P}_{\text {virE }}::$ pemK and

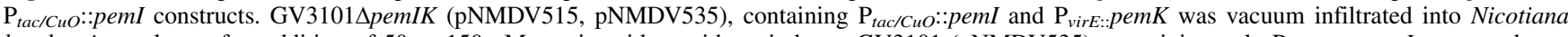
benthamiana plants after addition of 50 or $150 \mu \mathrm{M}$ cumic acid or without inducer. GV3101 (pNMDV535), containing only $\mathrm{P}_{\text {tac/CuO }}:: p e m I$, was used as a positive T-DNA transfer control. GFP loci were counted on agroinfiltrated leaves 6 days postinfiltration. Data are the mean \pm standard deviation of three independent experiments. Representative pictures of $N$. benthamiana leaves with GFP expression foci are seen on the top of the chart. 
mutagenesis of inserted genes or promoters. Only a combination of different strategies and further improvements of various systems can lead to the desired biosafety level.

\section{MATERIALS AND METHODS}

\section{Bacterial strains and growth conditions.}

Agrobacterium tumefaciens strains used in the study are listed in Table 2. E. coli strain DH5 $\alpha$ was used for all subclonings. Both E. coli and A. tumefaciens cells were grown in Luria Bertani (LB) medium at 37 and $28^{\circ} \mathrm{C}$, respectively. All media were supplemented, when necessary, with the following rates of antibiotics: $100 \mu \mathrm{g} / \mathrm{ml}$ ampicillin, $50 \mu \mathrm{g} / \mathrm{ml}$ spectinomycin, $50 \mu \mathrm{g} / \mathrm{ml}$ kanamycin, $50 \mu \mathrm{g} / \mathrm{ml}$ rifampicin, or $50 \mu \mathrm{g} / \mathrm{ml}$ nystatin. Restriction and modification enzymes from Thermo Fisher Scientific were used for all cloning steps.

\section{Construction of expression cassettes.}

PCR-amplified genes were cloned into pJET1.2/blunt or pTZ57R/T-commercial linearized positive-selection cloning vectors. Gene sequences were confirmed by sequencing and were inserted into binary expression vectors under the control of inducible promoters for induced expression in Agrobacterium strains. Constructed vectors were transferred into A. tumefaciens by electroporation.

A detailed description and schemes of plasmid construction are provided in Supplementary Figure S3, sequences of primers used in the study are listed in Supplementary Table S1, and relevant characteristics of plasmids are summarized in Supplementary Table S2.

\section{Plant growth conditions.}

Nicotiana benthamiana plants were grown under controlled conditions $\left(25^{\circ} \mathrm{C}\right.$ and $50 \%$ relative humidity) with a long-day photoperiod ( $16 \mathrm{~h}$ of light and $8 \mathrm{~h}$ of dark, with illumination of approximately 320 lux). Five- to six-week-old $N$. benthamiana plants were used for agroinfiltration experiments.

\section{Evaluation of the ability of toxins to induce death of A. tumefaciens ectopically.}

The overnight cultures of $A$. tumefaciens containing an inducible toxin were inoculated into $4 \mathrm{ml}$ of $\mathrm{LB}$ medium from frozen stock and were cultivated at $28^{\circ} \mathrm{C}$ in a shaker at $220 \mathrm{rpm}$. The optical density of o/n cultures were measured and cultures were diluted to an optical density at $595 \mathrm{~nm}\left(\mathrm{OD}_{595}\right)$ of approximately 0.1 to 0.2 . Each diluted culture was divided into two parts - the medium with an inducer [150 $\mu \mathrm{M}$ cumic acid (4isopropylbenzoic acid)] and the inducer-free medium. Induced and noninduced cultures were grown in the shaking incubator $\left(28^{\circ} \mathrm{C}, 220 \mathrm{rpm}\right)$ for $4 \mathrm{~h}$. Serial dilutions of induced and noninduced cultures were made and plated on the selective LB-agar medium without inducer. After two nights of growth, CFU of Agrobacterium were counted. The effectiveness of each toxin was evaluated by the following formula: $\Delta \log _{10}=\log _{10}(\mathrm{CFU} / \mathrm{ml}$ noninduced culture $)-\log _{10}(\mathrm{CFU} / \mathrm{ml}$ induced culture $)$. Raw data of the experiment are provided in Supplementary Table S3.

\section{Evaluation of GFP expression foci number and residual amount of agrobacterium after agro-spray transformation.}

Agrobacterium strains were inoculated from frozen stocks in $4 \mathrm{ml}$ of LB medium and were cultivated at $28^{\circ} \mathrm{C}$ with shaking at $220 \mathrm{rpm}$. Overnight cultures were diluted 1:1,000, starting from $\mathrm{OD}_{595}=1$ in tap water, and were supplemented with $0.005 \%$ Silwet L-77 and $150 \mu \mathrm{M}$ cumic acid if needed. Plants were sprayed five "sprays" to both sides of four upper leaves. GFP loci were counted on sprayed leaves 7 days after spraying, under UV light illumination. Next, the same four sprayed leaves were weighed and ground in liquid nitrogen for agrobacteria isolation and CFU enumeration. For every gram of fresh leaf, $3 \mathrm{ml}$ of sterile water was added, the plant tissue suspension was incubated for 30 to $40 \mathrm{~min}$ at room temperature, were filtrated through Miracloth, and then, $1 \mathrm{ml}$ was plated on the LB medium with the antibiotics rifampicin $(50 \mu \mathrm{g} / \mathrm{ml})$, kanamycin $(50 \mu \mathrm{g} / \mathrm{ml})$, spectinomycin $(50 \mu \mathrm{g} / \mathrm{ml})$, and nystatin $(50 \mu \mathrm{g} / \mathrm{ml})$, to ensure Agrobacterium growth and prevent the growth of other organisms. The amount of bacteria per gram of fresh leaves was calculated on plates after two nights of incubation in a $28^{\circ} \mathrm{C}$ incubator. Two plants were used for each experimental point. Three individual experiment repeats were made. Raw data of the experiment are provided in a Supplementary Table S4.

\section{Evaluation of GFP expression foci number after vacuum agroinfiltration.}

Agrobacterium strains were inoculated from frozen stocks in $4 \mathrm{ml}$ of LB medium and were cultivated at $28^{\circ} \mathrm{C}$ with shaking at $220 \mathrm{rpm}$. Overnight cultures were diluted 1:100,000, starting from $\mathrm{OD}_{595}=1$ in tap water, and were supplemented with $0.005 \%$ Silwet L-77 and 50 or $150 \mu \mathrm{M}$ cumic acid, if needed. Agrobacterium suspension was loaded into a desiccator vessel connected to a vacuum pump. The entire leaf system of a 5- to 6-week-old plant was then submerged into the suspension. Agroinfiltration was achieved by applying (till pressure of 200 mbar) and releasing vacuum through the pump. At 6 days after agroinfiltration, four infiltrated leaves from every plant were detached and GFP loci were counted under UV light illumination. Two plants were used for each experimental point. Three individual experiment repeats were made. Raw data of the experiment are provided in a Supplementary Table S5.

\section{VirE2 expression evaluation by reverse transcription- quantitative PCR (RT-qPCR).}

Agrobacterium strains with cumic acid-inducible promoters ICH011 (pNMDV386) and ICH011 (pNMDV356) and strain GV3101 (pICH27566) (Table 2) for wild-type virE2 expression evaluation were syringe-infiltrated into $N$. benthamiana plants as described by Denkovskiene et al. (2015). Cumic acid $(150 \mu \mathrm{M})$ was added for induced expression of virE2. Infiltrated plant leaves were harvested 4, 24, 48, and $72 \mathrm{~h}$ after agroinfiltration, and leaves were frozen in liquid nitrogen and were stored at $-70^{\circ} \mathrm{C}$ till RNA extraction. Plant tissue samples were ground in liquid nitrogen and total RNA was extracted, using Thermo Scientific GeneJET RNA purification kit. Thermo Scientific Verso 1-Step qRT-PCR kit SYBR green was used for qRT-PCR reactions. Reactions were performed according to given recommendations in a Corbett Rotor gene 6000 thermocycler. Expression ratio of virE2 from induced and noninduced promoters $\mathrm{P}_{t a c / C u O}, \mathrm{P}_{T 5 / C u O}$, and a native promoter of virE region were evaluated. RT-qPCR data were normalized to the expression of two reference genes, $r p o B$ and $16 s R N A$, as they showed a high and stable expression in A. tumefaciens. Primers used for reactions are listed in Supplementary Table S1. Reactions were repeated in triplicate.

\section{Construction of GV3101వpemIK strain.}

The region of pemIK was PCR-amplified together with upstream and downstream sequences from A. tumefaciens GV3101 genomic DNA (primers used can be found in Supplementary Table S1) and were inserted into a pJET1.2/blunt vector (Thermo Fisher scientific), resulting in a pNMDV90 construct. pNMDV90 was digested with $B g l \mathrm{II}-\mathrm{XbaI}$, and a fragment was ligated into vector pDNR1 (Clontech) digested with $B g l \mathrm{II}-N h e \mathrm{I}$. The resulting plasmid pNMDV421 was digested with MssI-NdeI 
(removing the coding sequence for pemIK) and re-circularized. The final plasmid pNMDV426 contains the $s a c B$ gene for counterselection on sucrose-containing medium. The construct pNMDV426 was transformed into A. tumefaciens GV3101 (list of constructed vectors can be found in Supplementary Tables S2 and S3). The pemIK genes were deleted by two homologous recombination events as described earlier (Berger and Christie 1993). The deletion of the pemIK region was confirmed by PCR and Southern blot analyses. The resulting Agrobacterium strain was named GV3101دpemIK.

\section{AUTHOR-RECOMMENDED INTERNET RESOURCE}

The RASTA-Bacteria tool: http://genoweb1.irisa.fr/duals/RASTA-Bacteria

\section{LITERATURE CITED}

Arcus, V. L., McKenzie, J. L., Robson, J., and Cook, G. M. 2011. The PINdomain ribonucleases and the prokaryotic VapBC toxin-antitoxin array. Protein Eng. Des. Sel. 24:33-40.

Bakar, F. A., Yeo, C. C., and Harikrishna, J. A. 2015. Expression of the Streptococcus pneumoniae yoeB chromosomal toxin gene causes cell death in the model plant Arabidopsis thaliana. BMC Biotechnol. $15: 26$.

Berger, B. R., and Christie, P. J. 1993. The Agrobacterium tumefaciens virB4 gene product is an essential virulence protein requiring an intact nucleoside triphosphate-binding domain. J. Bacteriol. 175:1723-1734.

Chan, C. T. Y., Lee, J. W., Cameron, D. E., Bashor, C. J., and Collins, J. J. 2016. 'Deadman' and 'Passcode' microbial kill switches for bacterial containment. Nat. Chem. Biol. 12:82-86.

Cho, H., and Winans, S. C. 2007. TraA, TraC and TraD autorepress two divergent quorum-regulated promoters near the transfer origin of the Ti plasmid of Agrobacterium tumefaciens. Mol. Microbiol. 63:17691782

Christensen, S. K., Maenhaut-Michel, G., Mine, N., Gottesman, S., Gerdes, K., and Van Melderen, L. 2004. Overproduction of the Lon protease triggers inhibition of translation in Escherichia coli: Involvement of the yefM-yoeB toxin-antitoxin system. Mol. Microbiol. 51:1705-1717.

de la Cueva-Méndez, G., Mills, A. D., Clay-Farrace, L., Díaz-Orejas, R., and Laskey, R. A. 2003. Regulatable killing of eukaryotic cells by the prokaryotic proteins Kid and Kis. EMBO J. 22:246-251

Denkovskiene, E., Paškevičius, Š., Werner, S., Gleba, Y., and Ražanskiene, A. 2015. Inducible expression of Agrobacterium virulence gene virE2 for stringent regulation of T-DNA transfer in plant transient expression systems. Mol. Plant-Microbe Interact. 28:1247-1255.

Fadeel, B., and Orrenius, S. 2005. Apoptosis: A basic biological phenomenon with wide-ranging implications in human disease. J. Intern. Med. 258:479-517.

Goeders, N., and Van Melderen, L. 2013. Toxin-antitoxin systems as multilevel interaction systems. Toxins (Basel). 10.3390/toxins6010304

Hahn, S., Giritch, A., Bartels, D., Bortesi, L., and Gleba, Y. 2015. A novel and fully scalable Agrobacterium spray-based process for manufacturing cellulases and other cost-sensitive proteins in plants. Plant Biotechnol. J. 13:708-716

Hodgins, B., Pillet, S., Landry, N., and Ward, B. J. 2019. A plant-derived VLP influenza vaccine elicits a balanced immune response even in very old mice with co-morbidities. PLoS One 14:e0210009.

Hu, M. X., Zhang, X., Li, E. L., and Feng, Y.-J. 2010. Recent advancements in toxin and antitoxin systems involved in bacterial programmed cell death. Int. J. Microbiol. 2010:781430.

Koncz, C., and Schell, J. 1986. The promoter of TL-DNA gene 5 controls the tissue-specific expression of chimaeric genes carried by a novel type of Agrobacterium binary vector. Mol. Gen. Genet. 204:383-396.

Krenek, P., Samajova, O., Luptovciak, I., Doskocilova, A., Komis, G., and Samaj, J. 2015. Transient plant transformation mediated by Agrobacterium tumefaciens: Principles, methods and applications. Biotechnol. Adv. 33:1024-1042.

Kristoffersen, P., Jensen, G. B., Gerdes, K., and Piškur, J. 2000. Bacterial toxin-antitoxin gene system as containment control in yeast cells. Appl. Environ. Microbiol. 66:5524-5526.

Lajoie, M. J., Rovner, A. J., Goodman, D. B., Aerni, H. R., Haimovich, A. D., Kuznetsov, G., Mercer, J. A., Wang, H. H., Carr, P. A., Mosberg, J. A., Rohland, N., Schultz, P. G., Jacobson, J. M., Rinehart, J., Church,
G. M., and Isaacs, F. J. 2013. Genomically recoded organisms expand biological functions. Science 342:357-360.

Lee, J. W., Chan, C. T. Y., Slomovic, S., and Collins, J. J. 2018. Nextgeneration biocontainment systems for engineered organisms. Nat. Chem. Biol. 14:530-537

Lee, M. W., Rogers, E. E., and Stenger, D. C. 2012. Xylella fastidiosa plasmid-encoded PemK toxin is an endoribonuclease. Phytopathology 102:32-40.

Mandell, D. J., Lajoie, M. J., Mee, M. T., Takeuchi, R., Kuznetsov, G., Norville, J. E., Gregg, C. J., Stoddard, B. L., and Church, G. M. 2015 Biocontainment of genetically modified organisms by synthetic protein design. Nature 518:55-60.

McCullen, C. A., and Binns, A. N. 2006. Agrobacterium tumefaciens and plant cell interactions and activities required for interkingdom macromolecular transfer. Annu. Rev. Cell Dev. Biol. 22:101-127.

Mutschler, H., and Meinhart, A. 2011. $\varepsilon / \zeta$ systems: Their role in resistance, virulence, and their potential for antibiotic development. J. Mol. Med. (Berl.) 89:1183-1194.

Sala, A., Bordes, P., and Genevaux, P. 2014. Multiple toxin-antitoxin systems in Mycobacterium tuberculosis. Toxins (Basel) 6:1002-1020.

Schulz, S., Stephan, A., Hahn, S., Bortesi, L., Jarczowski, F., and Bettmann, U., Paschke, A. K., Tusé, D., Stahl, C. H., Giritch, A., and Gleba, Y 2015. Broad and efficient control of major foodborne pathogenic strains of Escherichia coli by mixtures of plant-produced colicins. Proc. Natl. Acad. Sci. U.S.A. 112: E5454-E5460.

Sevin, E. W., and Barloy-Hubler, F. 2007. RASTA-Bacteria: A web-based tool for identifying toxin-antitoxin loci in prokaryotes. Genome Biol. 8:R155.

Szwacka, M., Burza, W., Zawirska-Wojtasiak, R., Góslínski, M., Twardowska, A., Gajc-Wolska, J., Kosieradzka, I., and Kiełkiewicz, M. 2012. Genetically modified crops expressing 35S-thaumatin II transgene: Sensory properties and food safety aspects. Comp. Rev. Food Sci. F. 11:174-186.

Tan, Q., Awano, N., and Inouye, M. 2010. YeeV is an Escherichia coli toxin that inhibits cell division by targeting the cytoskeleton proteins, FtsZ and MreB. Mol. Microbiol. 79:109-118.

Torisky, R. S., Kovacs, L., Avdiushko, S., Newman, J. D., Hunt, A. G., and Collins, G. B. 1997. Development of a binary vector system for plant transformation based on the supervirulent Agrobacterium tumefaciens strain Chry5. Plant Cell Rep. 17:102-108.

Tschofen, M., Knopp, D., Hood, E., and Stöger, E. 2016. Plant molecular farming: Much more than medicines. Annu. Rev. Anal. Chem. (Palo Alto, Calif.) 9:271-294.

Tsuchimoto, S., Nishimura, Y., and Ohtsubo, E. 1992. The stable maintenance system pem of plasmid R100: Degradation of PemI protein may allow PemK protein to inhibit cell growth. J. Bacteriol. 174 4205-4211.

Tusé, D., Tu, T., and McDonald, K. A. 2014. Manufacturing economics of plant-made biologics: Case studies in therapeutic and industrial enzymes. BioMed Res. Int. 2014:256135.

Wang, X., Lord, D. M., Cheng, H. Y., Osbourne, D. O., Hong, S. H., Sanchez-Torres, V., Quiroga, C., Zheng, K., Herrmann, T., Peti, W., Benedik, M. J., Page, R., and Wood, T. K. 2012. A new type V toxinantitoxin system where mRNA for toxin GhoT is cleaved by antitoxin GhoS. Nat. Chem. Biol. 8:855-861.

Wang, X., and Wood, T. K. 2011. Toxin-antitoxin systems influence biofilm and persister cell formation and the general stress response. Appl. Environ. Microbiol. 77:5577-5583.

Weel-Sneve, R., Kristiansen, K. I., Odsbu, I., Dalhus, B., Booth, J., Rognes, T., Skarstad, K., and Bjørås, M. 2013. Single transmembrane peptide DinQ modulates membrane-dependent activities. PLoS Genet. 9:e1003260.

Winther, K., Tree, J. J., Tollervey, D., and Gerdes, K. 2016. VapCs of Mycobacterium tuberculosis cleave RNAs essential for translation. Nucleic Acids Res. 44:9860-9871.

Winther, K. S., and Gerdes, K. 2011. Enteric virulence associated protein VapC inhibits translation by cleavage of initiator tRNA. Proc. Natl. Acad. Sci. U.S.A. 108:7403-7407.

Yamaguchi, Y., and Inouye, M. 2016. Toxin-antitoxin systems in bacteria and archaea. Chapter 2.7 in:Stress and Environmental Regulation of Gene Expression and Adaptation in Bacteria. F. J. de Bruijn, ed. John Wiley \& Sons, New York.

Yamamoto, S., Kiyokawa, K., Tanaka, K., Moriguchi, K., and Suzuki, K. 2009. Novel toxin-antitoxin system composed of serine protease and AAA-ATPase homologues determines the high level of stability and incompatibility of the tumor-inducing plasmid pTiC58. J. Bacteriol. 191: 4656-4666

Yao, J., Weng, Y., Dickey, A., and Wang, K. Y. 2015. Plants as factories for human pharmaceuticals: Applications and challenges. Int. J. Mol. Sci. 16:28549-28565. 